\title{
Long-Term Outcome of Distal Cholangiocarcinoma after Pancreaticoduodenectomy Followed by Adjuvant Chemoradiotherapy: A 15-Year Experience in a Single Institution
}

\author{
Byoung Hyuck Kim, MD',2 \\ Kyubo Kim, MD, PhD ${ }^{3}$ \\ Eui Kyu Chie, MD, PhD' \\ Jeanny Kwon, MD, PhD',4 \\ Jin-Young Jang, MD, $P h D^{5}$ \\ Sun Whe Kim, MD, PhD \\ Do-Youn Oh, MD, PhD ${ }^{6}$ \\ Yung-Jue Bang, $\mathrm{MD}, \mathrm{PhD}^{6}$
}

\section{${ }^{1}$ Department of Radiation Oncology, Seoul National University College of Medicine, Seoul, ${ }^{2}$ Division of Biological Warfare Preparedness and Response, Armed Forces Medical Research Institute, Daejeon, ${ }^{3}$ Department of Radiation Oncology, Ewha Womans University School of Medicine, Seoul, ${ }^{4}$ Department of Radiation Oncology, Chungnam National University School of Medicine, Daejeon, Departments of ${ }^{5}$ Surgery and ${ }^{6}$ Internal Medicine, Seoul National University College of Medicine, Seoul, Korea}

\author{
Correspondence: Kyubo Kim, MD, PhD \\ Department of Radiation Oncology, \\ Ewha Womans University School of Medicine, \\ 1071 Anyangcheon-ro, Yangcheon-gu, \\ Seoul 07985, Korea \\ Tel: 82-2-2650-5334 \\ Fax: 82-2-2654-0363 \\ E-mail: kyubokim.ro@gmail.com \\ Received April 20, 2016 \\ Accepted August 9, 2016 \\ Published Online August 23, 2016
}

\begin{abstract}
Purpose
This study was conducted to evaluate the long-term outcome in patients undergoing pancreaticoduodenectomy (PD) followed by adjuvant chemoradiotherapy for distal cholangiocarcinoma (DCC) in a high-volume center and to identify the prognostic impact of clinicopathologic factors.
\end{abstract}

\section{Materials and Methods}

A total of 132 consecutive patients who met the inclusion criteria were retrieved from the institutional database from January 1995 to September 2009. All patients received adjuvant treatments at a median of 45 days after the surgery. Median follow-up duration was 57 months (range, 6 to 225 months) for all patients and 105 months for survivors (range, 13 to 225 months).

\section{Results}

The 5-year locoregional recurrence-free survival (LRRFS), distant metastasis-free survival (DMFS), disease-free survival (DFS), and overall survival (OS) rates were 70.7\%, 55.7\%, $49.4 \%$, and $48.1 \%$, respectively. Univariate analysis revealed poorly differentiated (P/D) tumors and lymph node (LN) metastasis were significantly associated with DMFS and OS. Additionally, preoperative carbohydrate antigen 19-9 level was significantly correlated with DFS, LRRFS, and DMFS. Upon multivariate analysis for OS, P/D tumors $(p=0.015)$ and LN metastasis $(p=0.003)$ were significant prognosticators that predicted inferior OS. Grade 3 or higher late gastrointestinal toxicity occurred in only one patient $(0.8 \%)$.

\section{Conclusion}

Adjuvant chemoradiotherapy after PD for DCC is an effective and tolerable strategy without significant side effects. During long-term follow-up, we found that prognosis of DCC was mainly influenced by histologic differentiation and LN metastasis. For patients with these risk factors, further research should focus on improving adjuvant strategies as well as other treatment approaches.

\section{Introduction}

Extrahepatic bile duct (EHBD) cancer is an uncommon disease in Western countries; however, about 5,000 cases are newly diagnosed every year in Korea (crude incidence rate, 10.6 per 100,000 in 2013) [1]. Although there have been considerable advancements in surgical management and multi-
Key words

Distal cholangiocarcinoma, Pancreaticoduodenectomy, Adjuvant chemoradiotherapy 
to the application of postoperative radiotherapy. However, as we previously reported, our institution has consistently provided adjuvant chemoradiotherapy (CRT) for locoregionally advanced diseases (T2-4 or node positive) and microscopic residual disease (R1 resection) after surgery [8].

Tumor location is of clinical importance and may affect survival outcomes. Distal cholangiocarcinoma (DCC) is defined as bile duct tumors between the cystic duct and the ampulla of Vater (except Klatskin tumors and ampulla of Vater cancer), which include mid common bile duct tumors (between the junction with the cystic duct and the junction with the pancreas) and distal (intrapancreatic) bile duct tumors. DCC usually shows improved outcomes compared with perihilar tumors, which may be because of earlier clin- ical symptoms. Nevertheless, its prognosis remains poor. Moreover, the prognostic factors for these rare tumors have not been widely established because they only comprise about $30 \%$ of EHBD cancer occurrences [9,10]. Many previous studies showed weakness in heterogeneous treatments and insufficient follow-up duration [2-7]. Therefore, we evaluated the long-term outcome of patients undergoing PD followed by adjuvant CRT for DCC in a high-volume center to identify the prognostic impact of clinicopathological factors.

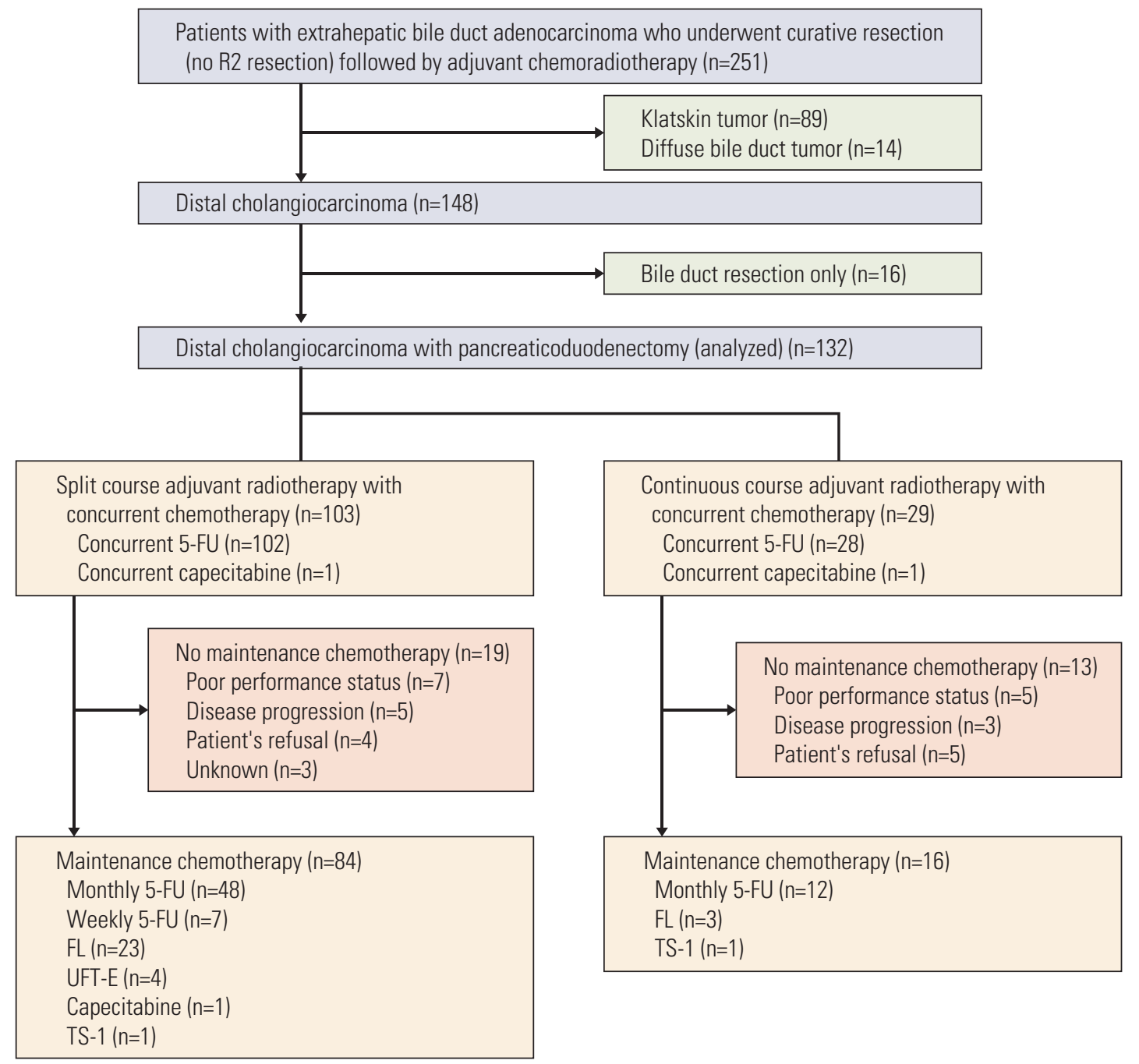

Fig. 1. CONSORT diagram of study patients. 5-FU, 5-fluorouracil; FL, 5-fluorouracil+leucovorin; UFT-E, enteric-coated tegafur/uracil. 


\section{Materials and Methods}

\section{Study population}

In our institution, most patients with resected EHBD cancer received adjuvant CRT (except those who had pT1N0 disease with $\mathrm{R} 0$ resection, poor performance status, or refused further treatment). Therefore, we searched for consecutive patients who underwent curative resection (except R2 resection) followed by adjuvant CRT for EHBD cancer from January 1995 to September 2009 in the institutional database of Seoul National University Hospital. Initially, 251 patients were found; however, 89 with Klatskin tumors, 14 with diffuse bile duct tumors, and 16 who only underwent bile duct resection were serially excluded (Fig. 1). As a result, a total of 132 DCC patients treated with PD followed by adjuvant CRT were eligible for this analysis. Institutional review board approval (H-1403-047-565) was obtained before data collection.

\section{Surgery and adjuvant treatments}

Resections included PD or pylorus-preserving PD, each of which were combined with regional lymph node (LN) dissection. TNM stage was re-evaluated during data collection according to the American Joint Committee on Cancer staging, seventh edition.

All patients received adjuvant treatments at a median of 45 days after surgery (range, 24 to 89 days). Preoperative chemotherapy or radiotherapy was not performed. In 103 patients, a total of $40 \mathrm{~Gy}$ in daily fractions of 2 Gy was delivered to the tumor bed and regional LNs (pericholedochal, retrocaval, and aortocaval). Patients had 2 weeks of planned rest after 20 Gy. Concomitant 5-fluorouracil (5-FU, $500 \mathrm{mg} /$ $\mathrm{m}^{2}$ / day intravenous bolus) was administered for the first 3 days of each 2 weeks of radiotherapy. A continuous course of radiotherapy was administered to 29 patients with a median dose of 50.4 Gy (range, 50 to 55.8 Gy) at 1.8-2.0 Gy per fraction. Concomitant fluoropyrimidine-based chemotherapy (intravenous $5-\mathrm{FU}, 500 \mathrm{mg} / \mathrm{m}^{2} /$ day or oral capecitabine) was administered during the first 3 days of the first and fifth week of radiotherapy. 5-FU-based maintenance chemotherapy was also administered to 100 patients after the completion of radiotherapy. Of the 100 patients, 60 patients received 5 -FU ( $500 \mathrm{mg} / \mathrm{m}^{2} /$ day) for 5 days every 4 weeks, 26 received 5-FU with leucovorin for 5 days every 4 weeks, seven received 5-FU ( $\left.500 \mathrm{mg} / \mathrm{m}^{2}\right)$ once weekly, four received enteric-coated tegafur/uracil, two were administered had TS-1, and one received oral capecitabine. Maintenance chemotherapy was not offered to 32 patients because of poor performance status after CRT $(n=12)$, patient refusal $(n=9)$, disease progression $(n=8)$, or for unknown reasons $(n=3)$.

\section{Assessment of recurrence and toxicity}

All patients were regularly followed up for surveillance for recurrence. Patient follow-up was completed by May 2014. Routine exams including appropriate imaging methods (abdomino-pelvic computed tomography [CT], ultrasonography, or magnetic resonance imaging) and serum tumor marker (carbohydrate antigen 19-9 [CA 19-9]) assessment were performed at prespecified intervals (typically every 3 months up to 2 years, followed by every 6 months) or when there were any suspicious findings suggestive of recurrence. Most recurrences were clinically diagnosed by imaging studies such as CT on a region of interest and/or positron emission tomography without pathologic confirmation. Recurrence patterns were classified as locoregional recurrence (LRR), distant metastasis (DM), or both. LRR was defined as recurrence in the tumor bed, anastomosis sites, or regional $\mathrm{LN}$ area. DM was defined as recurrence in the nonregional LN area or in other organs. Peritoneal seeding was determined by expert radiologists based on the results of CT analysis of ascites, thickening of the peritoneum (either smooth or nodular), or scalloping of the liver or splenic surface. Treatment toxicities caused by primary surgery or adjuvant CRT were also evaluated retrospectively through medical records. Gastrointestinal radiation toxicity was evaluated using the Radiation Therapy Oncology Group (RTOG) criteria.

\section{Statistical analyses}

Locoregional recurrence-free survival (LRRFS), distant metastasis-free survival (DMFS), disease-free survival (DFS), and overall survival (OS) were measured from the day of surgery to the time of LRR, DM, any failure or death from any causes, respectively, during the follow-up period. Patients who were alive or free of recurrence were censored at the time of the last follow-up. The actual survival rates were determined by the Kaplan-Meier method and compared using the log-rank test. The Cox proportional hazards model was used for multivariate analysis to estimate the hazard ratio and to adjust for potential confounding factors. Factors found to be significant upon univariate analysis or thought to be clinically relevant were subjected to both forward and backward stepwise selection multivariate analysis. A p-value of $<0.05$ was considered statistically significant. The differences in characteristics between groups were compared using a chi-square test. Data were analyzed using the SPSS ver. 18.0.1 (SPSS Inc., Chicago, IL). 
Table 1. Summary of patient characteristics

$\begin{array}{lr}\text { Characteristic } & \text { No. }(\%)(\mathbf{n}=\mathbf{1 3 2}) \\ \begin{array}{l}\text { Age (yr) } \\ \geq 60\end{array} & 82(62.1) \\ <60 & 50(37.9) \\ \text { Sex } & \\ \quad \text { Male } & 92(69.7) \\ \quad \text { Female } & 40(30.3) \\ \text { Performance (ECOG) } & \\ 0-1 & 111(84.1) \\ 2 & 21(15.9) \\ \text { Tumor location } & 65(49.2) \\ \quad \text { Mid common bile duct } & 67(50.8) \\ \text { Distal (intrapancreatic) bile duct } & \\ \text { Type of surgery } & 46(34.8) \\ \text { Pancreaticoduodenectomy } & 86(65.2) \\ \text { Pylorus-preserving } & \\ \text { pancreaticoduodenectomy } & \\ \text { Residual disease } & 118(89.4) \\ \text { R0 } & 14(10.6) \\ \text { R1 }\end{array}$

\section{Histologic differentiation}

$\mathrm{W} / \mathrm{D}, \mathrm{M} / \mathrm{D}$

$\mathrm{P} / \mathrm{D}$

Unknown 4 (3.0)

Tumor size (cm)

$\geq 2 \quad 90$ (68.2)

$<2 \quad 41(31.1)$

Unknown $1(0.7)$

Pathologic T stage

T1-2 $51(38.6)$

T3 81 (61.4)

Lymph node metastasis

$\begin{array}{ll}\text { Yes } & 43(32.6)\end{array}$

No $\quad 89(67.4)$

Overall stage

T1N0 $2(1.5)$

T1N1 $2(1.5)$

T2N0 $34(25.8)$

T2N1 $13(9.8)$

T3N0 $53(40.1)$

T3N1 28 (21.2)

Perineural invasion

Yes $\quad 103(78.0)$

No $28(21.2)$

Unknown $1(0.7)$

Preoperative CA19-9 (U/mL)

$\begin{array}{ll}\geq 37 & 71(53.8) \\ <37 & 35(26.5) \\ \text { Unknown } & 26(19.7)\end{array}$

ECOG, eastern cooperative oncology group; W/D, welldifferentiated; M/D, moderately-differentiated; P/D, poorly-differentiated; CA19-9, carbohydrate antigen 19-9.

\section{Results}

\section{Patient and tumor characteristics}

Overall patient and tumor characteristics are shown in Table 1 . The median age of all patients was 62 years (range, 34 to 77 years). Over half of all patients $(61.4 \%)$ had T3 disease; however, no patients had $\mathrm{T} 4$ disease. The median tumor size was $2 \mathrm{~cm}$ (range, 0.8 to $8.5 \mathrm{~cm}$ ). Resection margins were microscopically involved by invasive carcinoma in 14 patients $(10.6 \%)$, despite the absence of macroscopic residual lesions during PD. Pathologically, tumors were identified as well-differentiated (W/D) adenocarcinoma in 15 patients, moderately differentiated (M/D) adenocarcinoma in 94 patients, and poorly differentiated (P/D) adenocarcinoma in 19 patients. The median number of LNs dissected during surgery was 15 (range, 1 to 45). LN metastases were observed in 43 patients $(32.6 \%)$. In these patients, the median number of metastatic LNs was one (range, 1 to 9), and only nine patients had four or more LN metastases.

\section{Recurrence patterns and a case of delayed recurrence}

Median follow-up duration was 57 months (range, 6 to 225 months) for all patients and 105 months for survivors (range, 13 to 225 months). Overall, 66 patients (50.0\%) experienced recurrences. LRR occurred in 34 patients $(25.8 \%)$ and DM occurred in 58 patients $(43.9 \%)$. Time to recurrence was similar between patients with LRR (median, 13 months) and DM (median, 12 months). Only 13 patients (9.8\%) had an initial isolated local recurrence. In patients with DM, the most common site was the liver (30 patients, $51.7 \%$ ), followed by peritoneal seeding (18 patients, 31.0\%), nonregional LNs (three patients, $5.2 \%$ ), and others.

Of the 57 patients who did not develop recurrence during the first 5 years after surgery, only one developed late recurrence at 121 months after surgery. After a pylorus-preserving PD for mid common bile duct tumor (pT3N1M0-R1), she was disease-free for 121 months before LRR (hepaticojejunostomy site) and DM (liver and nonregional LN) were detected simultaneously. She refused further treatments and died 5 months later.

\section{Analysis of LRRFS, DMFS, DFS, and OS}

During the follow-up period, 89 patients $(67.4 \%)$ died. The 5-year LRRFS, DMFS, DFS, and OS rates were 70.7\%, 55.7\%, $49.4 \%$, and $48.1 \%$, respectively (Fig. 2). Among all patients, the 10-year OS was 34.1\% with 19 actual 10-year survivors $(14.4 \%)$. Among the group of recurred patients, median OS after recurrence was 7 months (range, 1 to 78 months). 
A
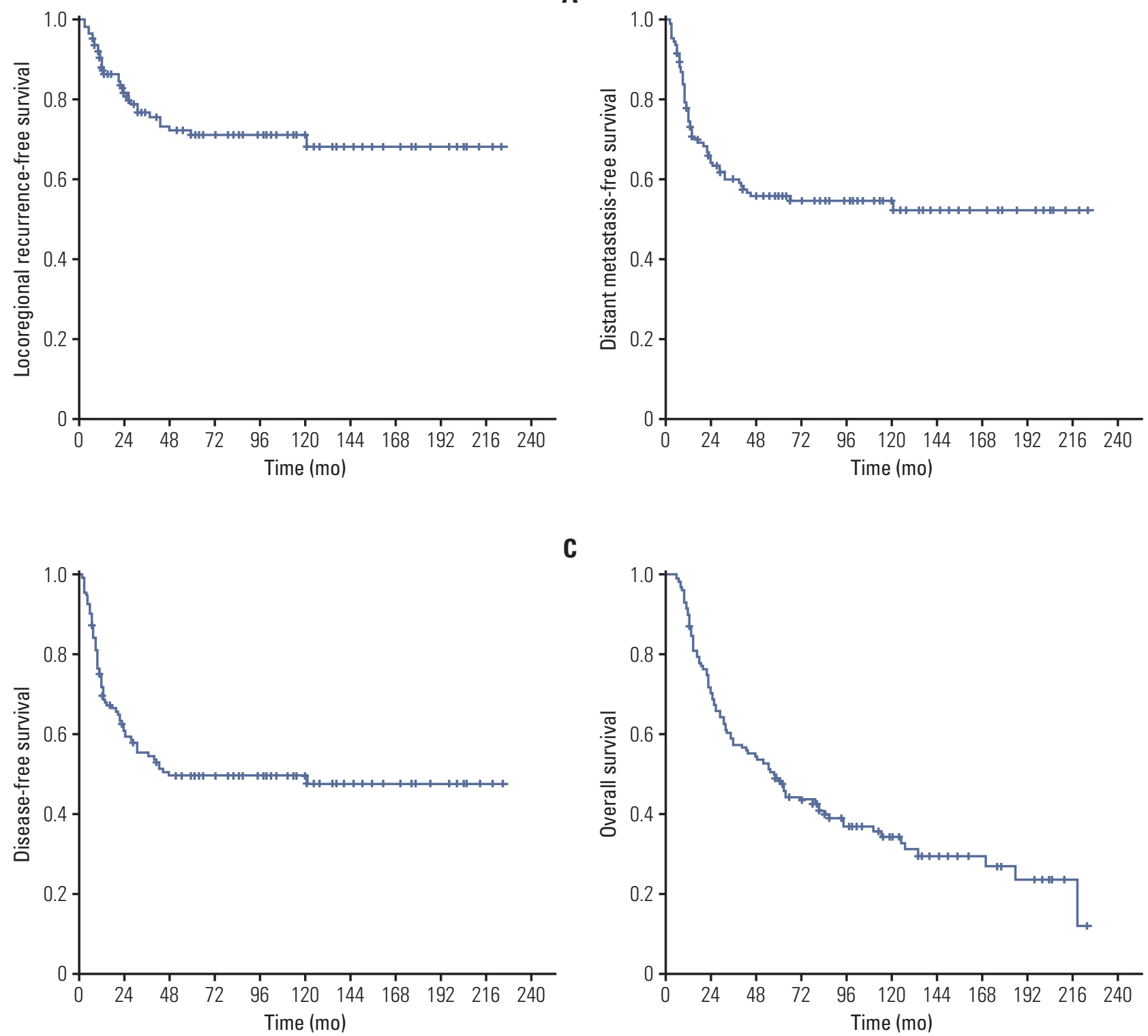

C

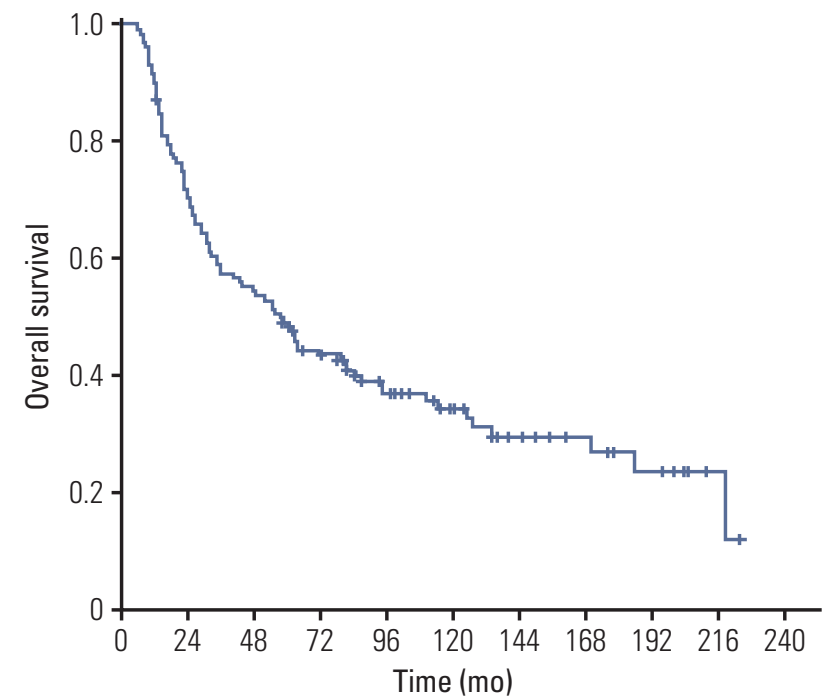

Fig. 2. Locoregional recurrence-free survival (A), distant metastasis-free survival (B), disease-free survival (C), and overall survival (D) in patients treated with pancreaticoduodenectomy followed by adjuvant chemoradiotherapy for distal cholangiocarcinoma.

The results of the univariate analyses of LRRFS, DMFS, DFS, and OS are shown in Table 2. Upon univariate analysis, $\mathrm{P} / \mathrm{D}$ tumors and LN metastasis were significantly associated with DMFS and OS, but not with LRRFS. Preoperative CA 19-9 level was also significantly correlated with DFS $(\mathrm{p}=0.014)$, LRRFS $(\mathrm{p}=0.006)$, and DMFS $(\mathrm{p}=0.032)$. R1 resection had marginal significance for LRRFS $(\mathrm{p}=0.077)$ and OS $(\mathrm{p}=0.067)$, but not for DMFS $(\mathrm{p}=0.312)$. Unusually, patients with poor performance status showed better survival out- comes without statistical significance (Table 2); however, this seemed due to several characteristic imbalances between the two groups (Supplementary Table 1).

Multivariate analysis incorporating residual disease, LN metastasis, and histologic differentiation demonstrated that $\mathrm{P} / \mathrm{D}$ tumors (hazard ratio [HR], 2.015; $\mathrm{p}=0.015$; backward selection) and LN metastasis (HR, 1.933; $\mathrm{p}=0.003$; backward selection) were significant prognosticators that predicted inferior OS (Table 3). The 5-year OS rates of patients with 


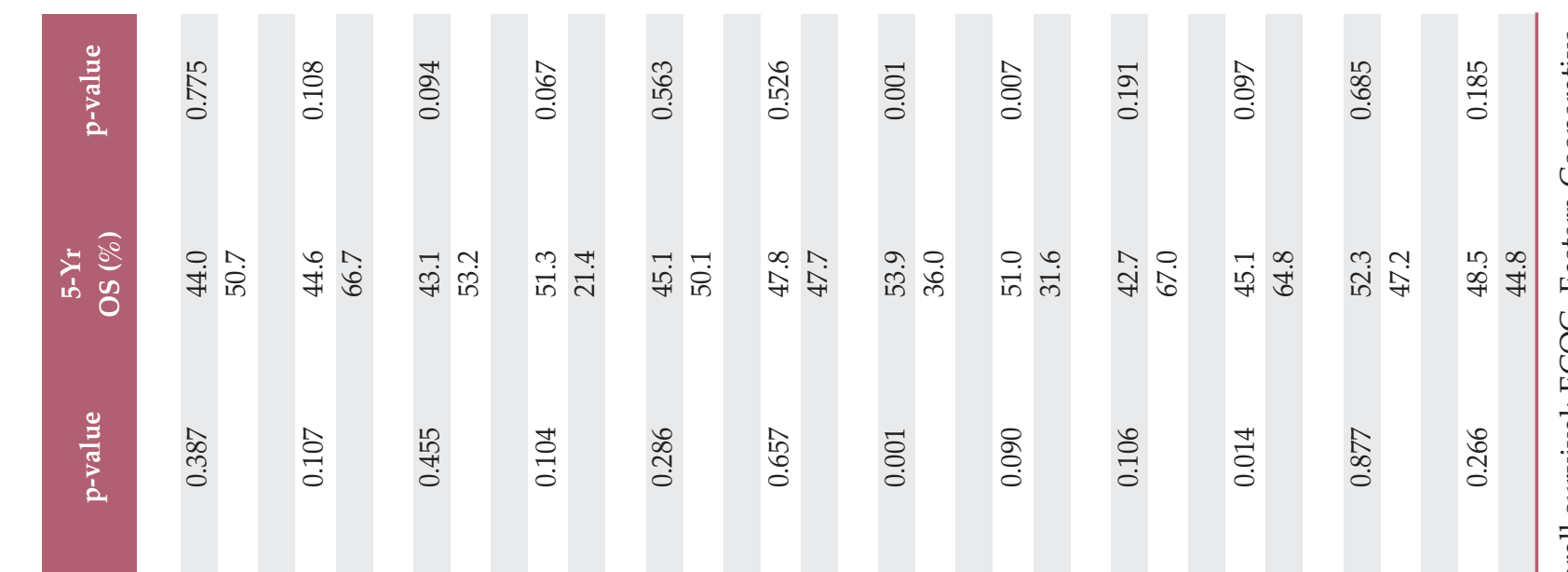

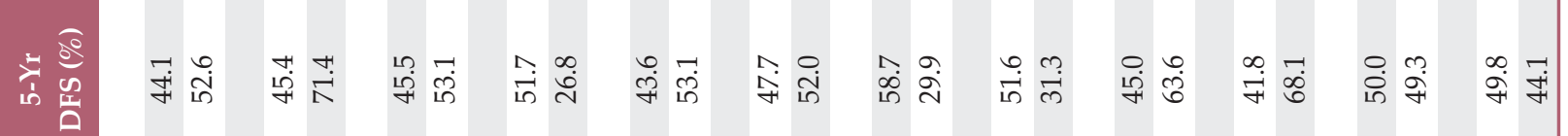

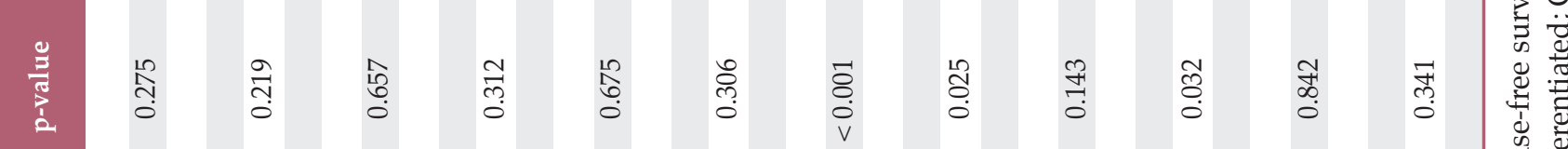

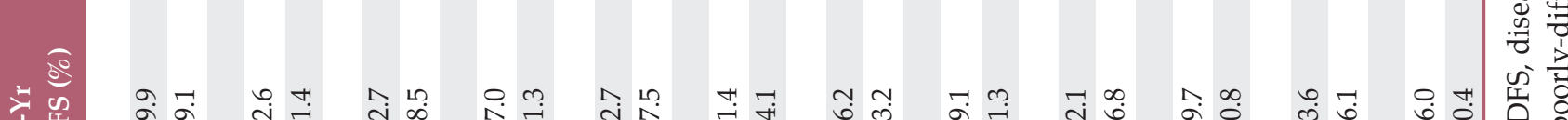

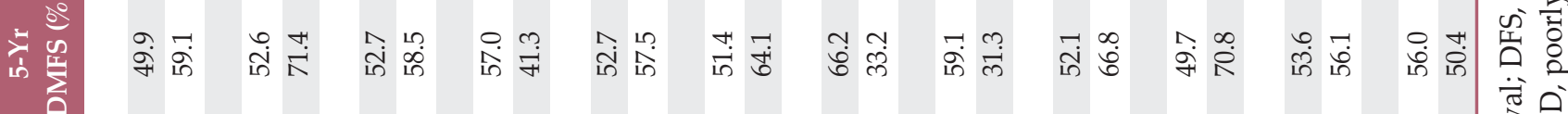

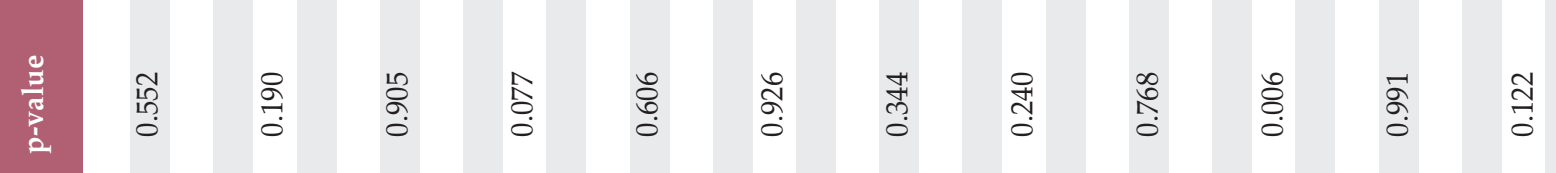

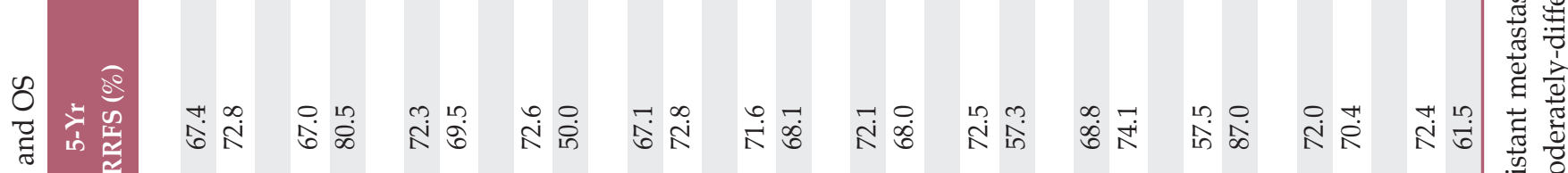

范

$\sum^{\infty}$

$\circ 2$

$\exists$

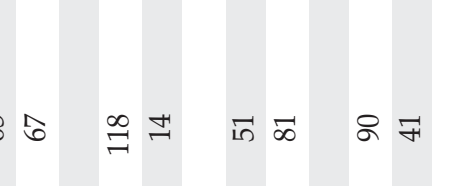

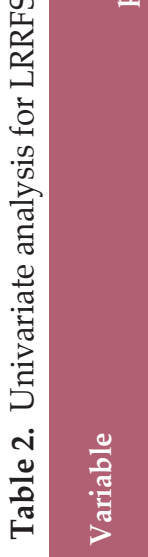
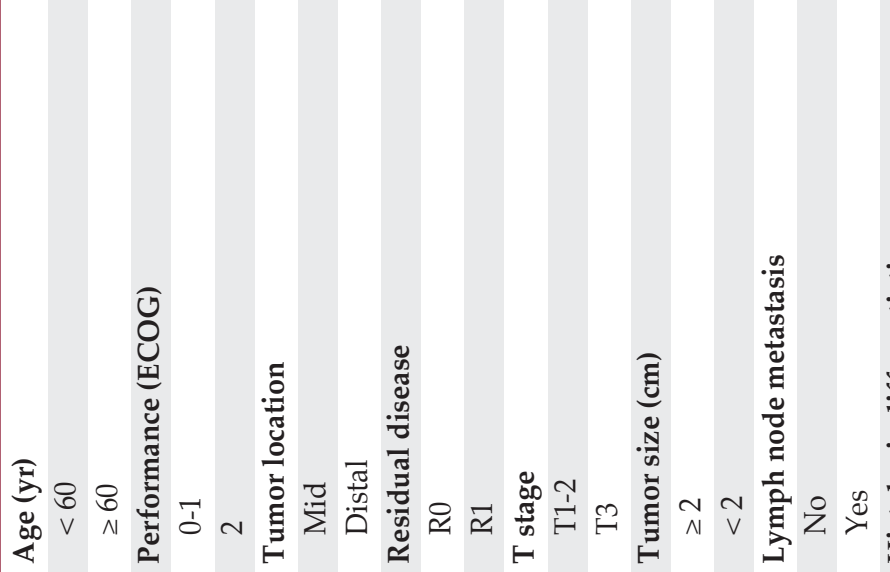

ஓ अ

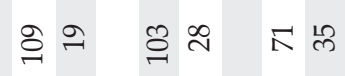

ㄱํㅇ \&

$\sum_{0}^{\infty} \frac{1}{\Sigma}$

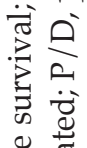

ఖँच

要

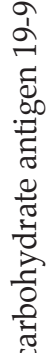

安

过

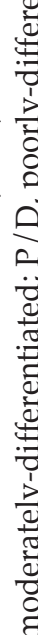

ते छे 
Table 3. Results of multivariate analysis of prognostic factors for the overall survival

\begin{tabular}{|c|c|c|c|c|c|c|}
\hline \multirow{2}{*}{ Variable } & \multicolumn{3}{|c|}{ Backward selection } & \multicolumn{3}{|c|}{ Forward selection } \\
\hline & Hazard ratio & $95 \% \mathrm{CI}$ & p-value & Hazard ratio & $95 \% \mathrm{CI}$ & p-value \\
\hline \multicolumn{7}{|c|}{ Histological differentiation } \\
\hline $\mathrm{W} / \mathrm{D}, \mathrm{M} / \mathrm{D}$ & 1 & & & 1 & & \\
\hline $\mathrm{P} / \mathrm{D}$ & 2.015 & $1.148-3.535$ & 0.015 & 1.865 & $1.073-3.241$ & 0.027 \\
\hline \multicolumn{7}{|c|}{ Lymph node metastasis } \\
\hline No & 1 & & & 1 & & \\
\hline Yes & 1.933 & $1.245-3.001$ & 0.003 & 1.951 & $1.257-3.031$ & 0.003 \\
\hline \multicolumn{7}{|c|}{ Residual disease } \\
\hline R0 & 1 & & & - & & \\
\hline R1 & 1.824 & $0.955-3.482$ & 0.069 & - & - & - \\
\hline
\end{tabular}

$\mathrm{CI}$, confidence interval; $\mathrm{W} / \mathrm{D}$, well-differentiated; $\mathrm{M} / \mathrm{D}$, moderately-differentiated; $\mathrm{P} / \mathrm{D}$, poorly-differentiated.

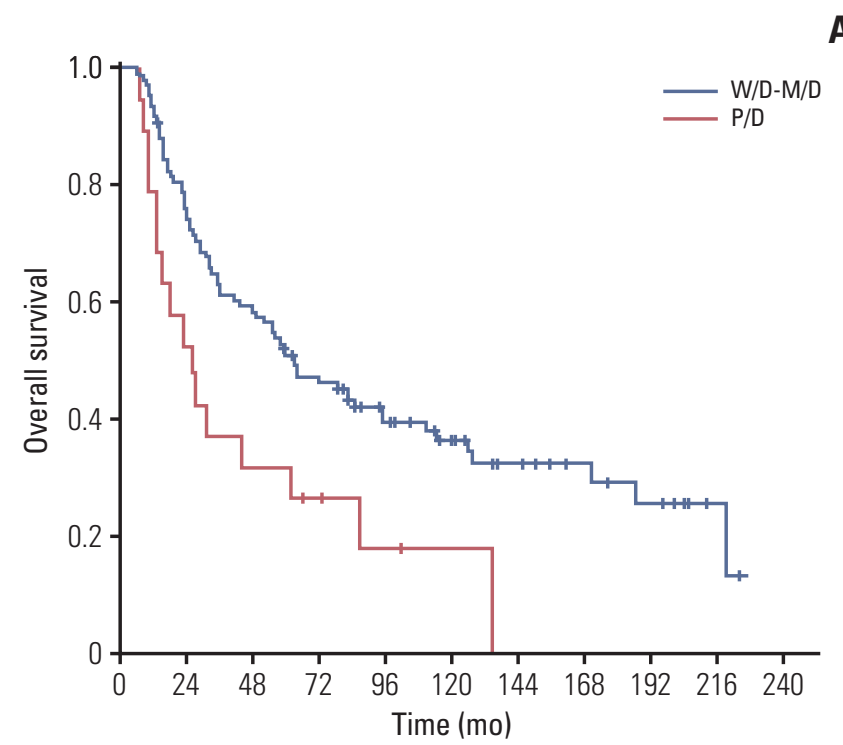

A

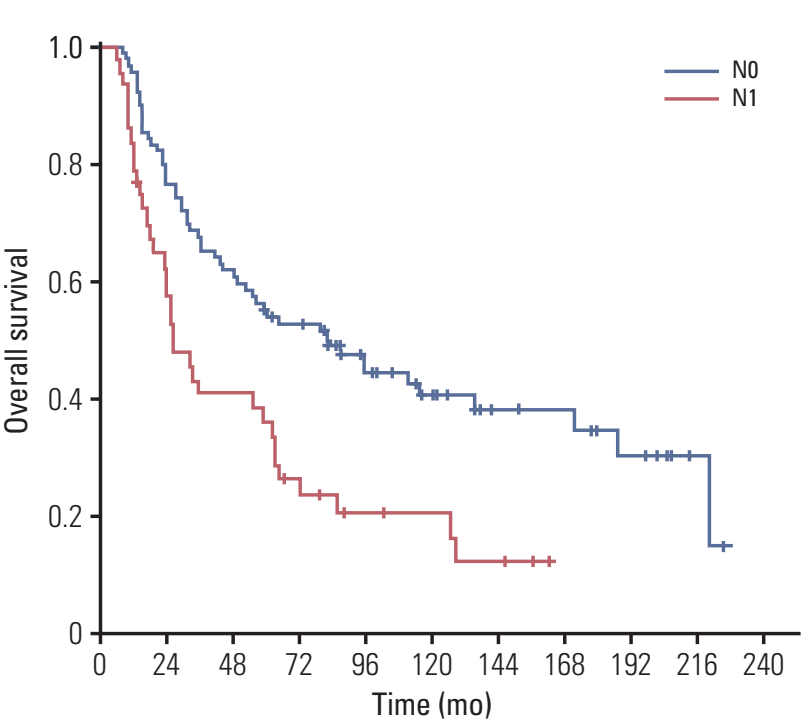

Fig. 3. Overall survival curves according to histologic differentiation (A) and $N$ stage (B). W/D, well-differentiated; $M / D$, moderately differentiated; $\mathrm{P} / \mathrm{D}$, poorly differentiated.

$\mathrm{W} / \mathrm{D}-\mathrm{M} / \mathrm{D}$ tumors and $\mathrm{P} / \mathrm{D}$ tumors were $51.0 \%$ and $31.6 \%$, respectively $(\mathrm{p}=0.007)$ (Fig. 3A). The 5-year OS rates of patients with N0 and N1 disease were $53.9 \%$ and $36.0 \%$, respectively ( $\mathrm{p}=0.001$ ) (Fig. 3B). Multivariate analysis of $\mathrm{LN}$ metastasis, preoperative CA 19-9 level, and histologic differentiation for DMFS revealed that LN metastasis was the only significant prognosticator that predicted inferior DMFS (HR, 2.345; $\mathrm{p}=0.005$; backward selection) (Fig. 4), while preoperative CA 19-9 level and histologic differentiation had borderline significance (Table 4). In addition, multivariate analysis of LRRFS including preoperative CA 19-9 level and residual disease revealed that preoperative CA 19-9 (HR, 3.898; $\mathrm{p}=0.011$; backward selection) was an independent adverse predictor, whereas $\mathrm{R} 1$ resection was not $(\mathrm{p}=0.305)$.

\section{Treatment toxicities}

Treatment toxicities were also comprehensively evaluated using retrospective medical records. Following PD, the most common complication was delayed wound healing (seven patients, 5.3\%), although stomy site leakage (four patients), fluid collection (three patients), postoperative cholangitis 


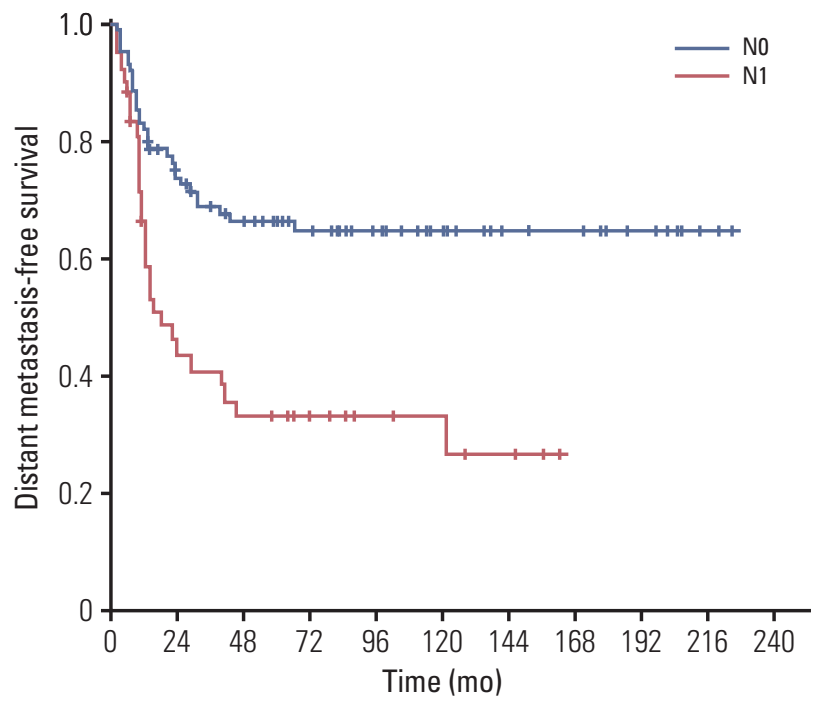

Fig. 4. Distant metastasis-free survival curves according to $\mathrm{N}$ stage.

(two patients), intra-abdominal abscess (two patients), postoperative bleeding (one patient), and postoperative pancreatitis (one patient) were also observed.

During CRT, acute gastrointestinal toxicity occurred in 88 patients (66.7\%), with RTOG grade 1 being observed in 37 (28.0\%) and grade 2 in $51(38.6 \%)$ patients. The most common acute symptoms were nausea and abdominal pain, which were tolerable and alleviated after supportive treatments. Grade 3 or higher toxicity did not occur during CRT. During follow-up, only one patient $(0.8 \%)$ experienced grade 3 late gastrointestinal toxicity. This patient underwent small bowel resection because of an adhesive ileus at 43 months after the end of a radiotherapy session, without evidence of LRR.

\section{Discussion}

This retrospective study was conducted to evaluate the long-term outcome of DCC in patients who underwent PD followed by adjuvant CRT. Although our study only analyzed a selected group of patients, the results suggest that adjuvant CRT for DCC following PD is an effective and tolerable treatment strategy without significant side effects.

As expected, long-term survival was still unsatisfactory; nevertheless, our finding of a 5 -year OS rate of $48.1 \%$ was comparable or slightly better than the results of previously reported studies [2-5,7,11]. Tumor differentiation and LN metastases were identified as important predictors of survival in our study, as demonstrated in other studies $[2-5,7,12]$. In particular, a meta-analysis conducted by Zhou et al. [13] revealed that LN metastasis was associated with shorter OS (risk ratio, 2.35; 95\% confidence interval, 1.89 to 2.93; $\mathrm{p}<0.0001$ ). Most studies included in this meta-analysis reported that LN metastasis has a powerful negative effect on survival. In our study, the frequency of LN metastasis was $32.6 \%$, and patients with LN metastasis had worse survival rates than those without it (5-year OS, $53.9 \%$ vs. $36.0 \%$; $\mathrm{p}=0.001$ ). Node-positive disease appeared to be incurable even after adjuvant CRT; however, the LN-positive group in our study was relatively favorable, with only a median of one metastatic LN. The dissection of regional LNs in our patients (median number, 15) appears to be sufficient accord-

Table 4. Results of the multivariate analysis of prognostic factors of distant metastasis-free survival

\begin{tabular}{|c|c|c|c|c|c|c|}
\hline \multirow{2}{*}{ Variable } & \multicolumn{3}{|c|}{ Backward selection } & \multicolumn{3}{|c|}{ Forward selection } \\
\hline & Hazard ratio & $95 \% \mathrm{CI}$ & p-value & Hazard ratio & $95 \% \mathrm{CI}$ & p-value \\
\hline \multicolumn{7}{|c|}{ Histological differentiation } \\
\hline $\mathrm{W} / \mathrm{D}, \mathrm{M} / \mathrm{D}$ & 1 & & & - & & \\
\hline $\mathrm{P} / \mathrm{D}$ & 1.839 & $0.921-3.671$ & 0.084 & - & - & - \\
\hline \multicolumn{7}{|c|}{ Lymph node metastasis } \\
\hline No & 1 & & & 1 & & \\
\hline Yes & 2.345 & $1.289-4.267$ & 0.005 & 2.730 & $1.517-4.914$ & 0.001 \\
\hline \multicolumn{7}{|c|}{ Preoperative CA19-9 (U/mL) } \\
\hline$<37$ & 1 & & & - & & \\
\hline$\geq 37$ & 1.913 & 0.944-3.875 & 0.072 & - & - & - \\
\hline
\end{tabular}

$\mathrm{CI}$, confidence interval; W/D, well-differentiated; M/D, moderately-differentiated; P/D, poorly-differentiated; CA19-9, carbohydrate antigen 19-9. 
ing to a recent study that evaluated the optimal extent of LN dissection in cholangiocarcinoma and suggested that a total LN count of 11 or more be used as the threshold for recommendation of DCC [14]. Therefore, the number of metastatic LNs in our patients may not be an underestimated value. The poor outcome after surgery and adjuvant CRT suggests that approaches other than upfront surgery may need to be considered, especially in cases of $\mathrm{LN}$-positive disease at the time of preoperative work-up. Even after curative resection, there may be a need for more effective adjuvant strategies for patients with LN metastasis.

A positive resection margin has been considered as an adverse prognostic factor in some studies $[6,7,11,13]$; however, its prognostic impact has not been fully determined because of inconsistent results among studies and diverse definitions. Incidences of a positive resection margin in EHBD cancer after curative resection can vary from $10 \%$ to as high as $70 \%$, which can be explained by differences between surgeons regarding the principle of the operation or the definition of a positive resection margin, such as whether to include carcinoma in situ [15-19]. Despite the controversy, the effects of $\mathrm{R} 1$ resection on survival outcomes was not significant in our DCC patients who underwent adjuvant CRT. Previous studies of adjuvant CRT also demonstrated that a comparison between patients with $\mathrm{R} 0$ and $\mathrm{R} 1$ resection showed no significant differences in OS and LRRFS $[20,21]$. These findings corresponded with the results of our study. Therefore, adjuvant CRT may improve outcome with R1 resection in EHBD cancer and also in DCC.

Our study had a LRR rate of $25.8 \%$, lower than the reported range from $35 \%$ to $74 \%$ even after complete resection in the previous studies $[3,7,22,23]$, demonstrating the important role of radiotherapy in terms of locoregional control. However, the major pattern of failure was shifted to DM, as we previously reported for EHBD cancer patients who underwent adjuvant CRT [8]. Even after maintenance chemotherapy, the DM rate was too high, which worsened patient survival and quality of life. Conversely, in the era of targeted therapy, newly developed targeted agents and immunotherapeutic agents have shown some promising results $[24,25]$. Therefore, to treat DM, which is not well controlled by conventional chemotherapy, and to further improve treatment outcome of this lethal disease, our efforts should be focused on incorporating new chemotherapeutic agents in a combined perioperative approach with radiotherapy.

To the best of our knowledge, no randomized controlled trials have evaluated the role of adjuvant CRT in EHBD cancer and in DCC. Given the lack of evidence supporting the application of adjuvant CRT, our favorable long-term results could help researchers establish the application of adjuvant CRT. A recent multicenter study in Korea also demonstrated that adjuvant CRT was associated with significantly improved relapse-free survival and OS in patients with R0-resected DCC [26]. Moreover, our findings showed that acute gastrointestinal toxicity caused by adjuvant radiotherapy was mild, and that late toxicity (grade 3 or more) only occurred in one patient $(0.8 \%)$, even after a long-term followup. Other recent studies also reported that few cases of severe gastrointestinal complications were associated with radiotherapy [21,27]. Unlike perihilar tumors, radiosensitive adjacent organs such as the liver may be less affected by adjuvant radiotherapy in the case of DCC. Recent advances in radiotherapy techniques may further lower its unintended toxicity. However, further prospective trials are needed to evaluate the efficacy and safety of adjuvant CRT for DCC.

For a median follow-up of 57 months, about three-fifths of our patients had a recurrence but recurrence 5 years after surgery was rarely observed. These results were somewhat different from those of a previous study by Jang et al. [28], who reported that late recurrence after 5 years was not uncommon in EHBD cancer. However, about one-third of the patients in that study received hepatobiliary resection or bile duct resection for proximal EHBD tumor, while only one-third of the patients received adjuvant treatments. Unfortunately, further comparison of this aspect with other retrospective series was difficult because a limited number of studies have mentioned late recurrences after 5 years, possibly due to insufficient follow-up. Furthermore, some studies have suggested that late recurrence likely resulted from residual carcinoma in situ after resection, which had less malignancy and slower growth than invasive carcinoma $[19,29]$. However, the effects of residual carcinoma in situ on postoperative recurrence or survival have not been described in most studies and thus remain unclear. Accordingly, further studies of the characteristics of late recurrence after PD are required for the adequate management of DCC patients during a long-term follow-up.

It should be noted that there are several limitations to this study. Specifically, its retrospective nature might be a significant weakness; however, we thought that it would be difficult to conduct a prospective study because of the rarity of DCC. Moreover, although our institution offered adjuvant CRT to almost all patients who underwent PD, the patients evaluated in this study were selected ones who both underwent surgery and received adjuvant therapy. In addition, treatment-related toxicity might be underestimated because it was not considered prospectively. Finally, heterogeneous details of adjuvant CRT during a relatively long study period (for example, radiotherapy dose or type of maintenance chemotherapy) might also influence clinical outcomes. 


\section{Conclusion}

In conclusion, we reported the actual long-term outcome of DCC in patients who underwent PD followed by adjuvant CRT. During long-term follow-up, we found that prognosis of DCC after PD was mainly influenced by histologic differentiation and LN metastasis. For patients with these risk factors, further research should focus on improving adjuvant strategies as well as other treatment approaches.

\section{Electronic Supplementary Material}

Supplementary materials are available at Cancer Research and Treatment website (http:// www.e-crt.org).

\section{Conflicts of Interest}

Conflict of interest relevant to this article was not reported.

\section{Acknowledgments}

Byoung Hyuck Kim was supported by an institutional R\&D project funded by the Armed Forces Medical Research Institute (2015-AFMRI-02). Kyubo Kim was supported by the Ewha Womans University Research Grant of 2016.

\section{References}

1. Oh CM, Won YJ, Jung KW, Kong HJ, Cho H, Lee JK, et al. Cancer statistics in Korea: incidence, mortality, survival, and prevalence in 2013. Cancer Res Treat. 2016;48:436-50.

2. Andrianello S, Paiella S, Allegrini V, Ramera M, Pulvirenti A, Malleo G, et al. Pancreaticoduodenectomy for distal cholangiocarcinoma: surgical results, prognostic factors, and longterm follow-up. Langenbecks Arch Surg. 2015;400:623-8.

3. Koo TR, Eom KY, Kim IA, Cho JY, Yoon YS, Hwang DW, et al. Patterns of failure and prognostic factors in resected extrahepatic bile duct cancer: implication for adjuvant radiotherapy. Radiat Oncol J. 2014;32:63-9.

4. Kim HJ, Kim CY, Hur YH, Koh YS, Kim JC, Kim HJ, et al. Prognostic factors for survival after curative resection of distal cholangiocarcinoma: perineural invasion and lymphovascular invasion. Surg Today. 2014;44:1879-86.

5. Tan X, Xiao K, Liu W, Chang S, Zhang T, Tang H. Prognostic factors of distal cholangiocarcinoma after curative surgery: a series of 84 cases. Hepatogastroenterology. 2013;60:1892-5.

6. van der Gaag NA, Kloek JJ, de Bakker JK, Musters B, Geskus RB, Busch OR, et al. Survival analysis and prognostic nomogram for patients undergoing resection of extrahepatic cholangiocarcinoma. Ann Oncol. 2012;23:2642-9.

7. Kamposioras K, Anthoney A, Fernandez Moro C, Cairns A, Smith AM, Liaskos C, et al. Impact of intrapancreatic or extrapancreatic bile duct involvement on survival following pancreatoduodenectomy for common bile duct cancer. Br J Surg. 2014;101:89-99.

8. Kim K, Chie EK, Jang JY, Kim SW, Han SW, Oh DY, et al. Distant metastasis risk stratification for patients undergoing curative resection followed by adjuvant chemoradiation for extrahepatic bile duct cancer. Int J Radiat Oncol Biol Phys. 2012;84:81-7.

9. Miyakawa S, Ishihara S, Horiguchi A, Takada T, Miyazaki M, Nagakawa T. Biliary tract cancer treatment: 5,584 results from the Biliary Tract Cancer Statistics Registry from 1998 to 2004 in Japan. J Hepatobiliary Pancreat Surg. 2009;16:1-7.

10. Nakeeb A, Pitt HA, Sohn TA, Coleman J, Abrams RA, Piantadosi S, et al. Cholangiocarcinoma: a spectrum of intrahepatic, perihilar, and distal tumors. Ann Surg. 1996;224:463-73.

11. Kwon HJ, Kim SG, Chun JM, Lee WK, Hwang YJ. Prognostic factors in patients with middle and distal bile duct cancers. World J Gastroenterol. 2014;20:6658-65.

12. Wiltberger G, Krenzien F, Benzing C, Atanasov G, Klein F, Hau HM, et al. Prognostic accuracy of the seventh edition of the TNM classification compared with the fifth and sixth edition for distal cholangiocarcinoma. Ann Surg Oncol. 2016; 23:1320-6.

13. Zhou Y, Liu S, Wu L, Wan T. Survival after surgical resection of distal cholangiocarcinoma: a systematic review and metaanalysis of prognostic factors. Asian J Surg. 2017;40:129-38.

14. Ito K, Ito H, Allen PJ, Gonen M, Klimstra D, D'Angelica MI, et al. Adequate lymph node assessment for extrahepatic bile duct adenocarcinoma. Ann Surg. 2010;251:675-81.

15. Sakamoto Y, Shimada K, Nara S, Esaki M, Ojima H, Sano T, et al. Surgical management of infrahilar/suprapancreatic cholangiocarcinoma: an analysis of the surgical procedures, surgical margins, and survivals of 77 patients. J Gastrointest Surg. 2010;14:335-43.

16. Endo I, House MG, Klimstra DS, Gonen M, D'Angelica M, Dematteo RP, et al. Clinical significance of intraoperative bile 
duct margin assessment for hilar cholangiocarcinoma. Ann Surg Oncol. 2008;15:2104-12.

17. Wakai T, Shirai Y, Moroda T, Yokoyama N, Hatakeyama K. Impact of ductal resection margin status on long-term survival in patients undergoing resection for extrahepatic cholangiocarcinoma. Cancer. 2005;103:1210-6.

18. Sasaki R, Takeda Y, Funato O, Nitta H, Kawamura H, Uesugi $\mathrm{N}$, et al. Significance of ductal margin status in patients undergoing surgical resection for extrahepatic cholangiocarcinoma. World J Surg. 2007;31:1788-96.

19. Nakanishi Y, Kondo S, Zen Y, Yonemori A, Kubota K, Kawakami H, et al. Impact of residual in situ carcinoma on postoperative survival in 125 patients with extrahepatic bile duct carcinoma. J Hepatobiliary Pancreat Sci. 2010;17:166-73.

20. Kim K, Chie EK, Jang JY, Kim SW, Han SW, Oh DY, et al. Adjuvant chemoradiotherapy after curative resection for extrahepatic bile duct cancer: a long-term single center experience. Am J Clin Oncol. 2012;35:136-40.

21. Park JH, Choi EK, Ahn SD, Lee SW, Song SY, Yoon SM, et al. Postoperative chemoradiotherapy for extrahepatic bile duct cancer. Int J Radiat Oncol Biol Phys. 2011;79:696-704.

22. Kim JK, Ha HK, Han DJ, Auh YH. CT analysis of postoperative tumor recurrence patterns in periampullary cancer. Abdom Imaging. 2003;28:384-91.

23. de Castro SM, Kuhlmann KF, van Heek NT, Busch OR, Offer- haus GJ, van Gulik TM, et al. Recurrent disease after microscopically radical (R0) resection of periampullary adenocarcinoma in patients without adjuvant therapy. J Gastrointest Surg. 2004;8:775-84.

24. Oyasiji T, Zhang J, Kuvshinoff B, Iyer R, Hochwald SN. Molecular targets in biliary carcinogenesis and implications for therapy. Oncologist. 2015;20:742-51.

25. Marks EI, Yee NS. Immunotherapeutic approaches in biliary tract carcinoma: current status and emerging strategies. World J Gastrointest Oncol. 2015;7:338-46.

26. Kim YS, Hwang IG, Park SE, Go SI, Kang JH, Park I, et al. Role of adjuvant therapy after R0 resection for patients with distal cholangiocarcinoma. Cancer Chemother Pharmacol. 2016;77: 979-85.

27. Kim TH, Han SS, Park SJ, Lee WJ, Woo SM, Moon SH, et al. Role of adjuvant chemoradiotherapy for resected extrahepatic biliary tract cancer. Int J Radiat Oncol Biol Phys. 2011;81: e853-9.

28. Jang JY, Kim SW, Park DJ, Ahn YJ, Yoon YS, Choi MG, et al. Actual long-term outcome of extrahepatic bile duct cancer after surgical resection. Ann Surg. 2005;241:77-84.

29. Han IW, Jang JY, Lee KB, Kang MJ, Kwon W, Park JW, et al. Clinicopathological analysis and prognosis of extrahepatic bile duct cancer with a microscopic positive ductal margin. HPB (Oxford). 2014;16:575-81. 